\title{
MODELING DESIGN STUDIO PEDAGOGY: A MENTORED REFLECTIVE PRACTICE
}

\author{
J. Milovanovic ${ }^{1, \otimes}$ and J. Gero ${ }^{2}$ \\ ${ }^{1}$ UMR AUU-CRENAU, France, ${ }^{2}$ UNC Charlotte, United States of America \\ $\bigotimes$ julie.milovanovic@crenau.archi.fr
}

\begin{abstract}
This paper explores the notion of mentored reflective practice to describe design studio pedagogy. Students learn how to design by doing design in the studio. Design critiques are a key element in design studio pedagogy as they capture a moment where students get feedback from their tutors on their designs. The research questions addressed concern the roles of each participant during design studio reviews and their interactions with design representations. The protocol analysis methodology and the Function Behavior Structure ontology are utilized to convey our case study analysis.
\end{abstract}

Keywords: design education, co-design, design review, design representation, protocol analysis

\section{Introduction}

The design studio is finding its way into engineering education. It has been used extensively in architectural education as it is one of the cornerstones of the architecture curriculum. The aim of the design studio is for students to acquire design skills and to learn to think in a designerly way. Design studio pedagogy is project-based and also functions as a mentorship between tutors and students. Design is at the core of tutors' pedagogic strategies to teach design, where designing is either demonstrated, explained, observed or accompanied. The objective of the research described in this paper is to propose a model of the design studio pedagogy, that we call a mentored reflective practice. Our model is based on an existing design ontology, the Function Behavior Structure (FBS) (Gero, 1990).

Design critiques or reviews, held weekly in the design studios, form a key moment in the students' design learning experience. They include several steps: students present their designs, tutors give feedback on the designs and both discuss potential issues and solutions (Oh et al., 2013). Tutors are usually professionals without pedagogical training. With a better understanding of the design critique situation, we can provide feedback to design educators and define guidelines to follow to provide students with the most suitable design learning experiences.

In this paper, we study the evolution of the role of each participant (tutors and students) during three sequential architectural design critiques for each of three students, giving us 9 critique sessions. The design brief for this master studio is the development of a mixed use building, including housing units, offices and a social space, such as a shared garden, a library or a pub. The research questions addressed are as follows: What are the roles of each participant in the mentored reflective practice during design critiques? What are the participants' actions on the design representations? 


\section{Background}

\subsection{The design studio and design critiques}

The pedagogical methods used to teach design in the studios are diverse: theoretical courses, workshops, informal discussions, design critiques and juries. The main pedagogical objective of the design studio is to place students in a design situation. Tutors seek to share design knowledge and skills with students so that they develop the ability to design. Designing is based on cognitive processes influenced by previous design experiences. As a result, tutors are often unable to explicitly explain to students how they design and what is expected in terms of design. In the first pages of The Design Studio, Schön (1985) proposes a definition of the design studio as a prototype of collective and individual learning by doing, influenced and guided by the feedback of a practitioner.

The objectives of the critique are to evaluate the students' work, while providing constructive feedback on the design development. Empirical studies analyzing student/tutor design conversations in design critiques reveals the presence of design activities (Ferreira et al., 2016; Gero and Jiang, 2016) or verbal markers related to reasoning and reflection (Cardoso et al., 2014; Dong et al., 2016; Wolmarans, 2016). The content of the critiques depends on the studio tutors and guests during the juries. Some will become involved in specific design elements because they have expert knowledge of these elements (Barrett, 2000; Healy, 2016). The content of tutor's critiques is adjusted according to the pedagogical objectives, which may be centered on the design process or the concept, the quality of communication and representation, innovation with priority given to creativity or, on the contrary, an emphasis on functionalism (Goldschmidt et al., 2014).

In The Design Studio, Schön (1985) identifies four types of actions in design critiques: telling (tutor) and listening (student); demonstrating (tutor) and imitating (student). The first set corresponds to the explicit formulation of design knowledge, such as specific instructions to be followed, design theories, requirements concerning the format of representations or design references. The second set refers to a design situation through the tutor's demonstration (Goldschmidt et al., 2010; Heylighen et al., 1999; Schön, 1985).

\subsection{Importance of design representations}

In many design fields, a full-scale testing of the design is rarely possible, and the first prototype is the artifact delivered. Design involves and requires the manipulation of multiple types of representations at different scales related to the design's progress phases. Designers work simultaneously in a synthetic and analytical way (Lebahar, 1983), which explains their need to use a variety of representations of their design. The diversity of viewpoints and scales of representation allows them to deal with specific details in parallel with global concepts. Schön (1992) talks about designers' reflective conversations with the material of the design situation, which is the set of representations of the project, externalized by designers. Design knowledge is integrated into the external design representations, and its manipulation influences the direction taken during the design process. During design reviews, design representations serve as a communication tool, a design tool and also a pedagogic tool.

Sketches and diagrams are often used early in the design process (Purcell and Gero, 1998) and are commonly used by tutors during the critiques to express an idea. According to Ferguson (1992), drawing is used by the designer to try out different options, different ideas and has several functions: a thinking sketch, a prescriptive sketch and a talking sketch. Each projection (plans, sections) taken separately describes spatial characteristics of the design, which supports several levels of information to be integrated when all representations are considered. The fragmentation of design representations allows the designer to focus on a design element that appears in a specific type of representation, while maintaining his synthetic understanding of the whole design object (Estevez, 2001). This dissociation between different design representation brings a certain efficiency to the design process. The 3D representation synthesizes the spatial correspondence between plans, sections and elevations. It can offer an egocentric point of view on the design, from the pedestrian view point. According to Elsen and Heylighen (2014), pedestrian perspective representation provides an enhanced synthesis of design elements, represented in a fragmented way by plans and sections, and which includes a sensitive experience dimension of architecture. 


\section{Theoretical framework and methodology}

In the previous section, we pointed out that design critiques are centered on a learning by designing approach, where the tutor acts as a mentor for the student. Design representations support tutors' pedagogic strategies by providing an environment for tutors and students to interact in order to discuss and address design issues. In the following, we develop our model to describe this situation and the methodology used for our analysis.

\subsection{A mentored reflexive practice to describe design studio pedagogy}

We propose a theoretical framework to analyze design critiques in the studios: the mentored reflective practice. It is based on Schön's (1983) notion of reflective practice, which he defines as the designer's way of thinking, situated in the current action and in relation to their past experiences. Reflective practice encompasses tacit design processes used by designers to respond to a design situation by placing reflection at the core of that process. Schön (1992) specifies the importance of interactions between designers and their concepts during their reflective practice, which he defines as a reflective conversion with the materials of the design situation. The materials are the ensemble of externalized design representations and mental design representations, internal and personal to the designer. Reflective practice is the learning objective of the design studio, and, by its implicit nature, raises a real pedagogical challenge. The situatedness and implicitness of reflective practice imply that a specific pedagogical strategy, mentored-based, is suitable to teach design (Cross, 2006; Lawson and Dorst, 2009; Schön, 1985). The mentor combines theory and practice in the modeling of his expertise to the mentee (Dennen and Burner, 2008). The notion of mentored reflective practice that we propose defines the content of studio learning and the pedagogical approach used.

\subsection{Modeling the reflective practice}

Schön (1992) proposes a situated model of reflective practice emphasizing the interaction between the designer and the externalization of his/her concept through tangible, symbolic or iconic representations. The Function-Behavior-Structure ontology (Gero, 1990; Gero and Kannengiesser, 2004) proposes a descriptive model of the reflective practice, including the situatedness of designing at a personal level (self-reflection) and at a social level (reflection with the external design representations) (Gero and Kannengiesser, 2008). The reflective practice defined by Schön (1983) and represented in Gero and Kannengiesser's (2004) situated FBS model defines the situation of a single designer, and is extended to a co-design activity in the situated FBS co-design model (Gero and Milovanovic, 2019).

\subsubsection{The situated FBS ontology}

Here we provide a brief introduction to the situated FBS ontology followed by the situated FBS codesign model. For a full description consult Gero and Milovanovic (2019). The FBS ontology provides a description of design knowledge and design processes during a design activity. This ontology presents six design issues and eight design processes at the ontological level. Requirement $(\mathrm{R})$ include the design brief, client or regulation requirements. Function $(\mathrm{F})$ is the design object teleology, i.e. what the design object is for. Behaviors represent how the design object performs: it can be an expected behavior (Be) or a behavior derived from the structure of the design object (Bs). Structure (S) is the description of elements or groups of elements of the design object and their relationships. Description (D) represents externalizations representing the design object. Eight transformations from one issue to another describe design processes, Figure 1(a).

The notion of situatedness in design takes into account the past experiences and current information from the design environment (social) and the designer (personal). Each design situation is unique and each designer will react differently to it. The situated FBS framework is a cognitively articulated version of the FBS ontology that combines the FBS design processes with four cognitive processes: interpretation, constructive memory, focus and action (Gero and Kannengiesser, 2004). Three distinct worlds are identified in the situated FBS: the external world, the interpreted world and the expected world, which is part of the interpreted world, Figure 1(b). The external world holds all external representations of the design situation, verbal and graphic. It comprises all design issues of the FBS 
ontology (Requirement, Function, Behavior, Structure and Description). The interpreted world is the designer's construction of the design situation based on his/her perception, which is based on each individual's experience of the external world and his/her current design concepts. The expected world contains the formalization of possible design actions built upon the designer's interpreted world. It sits within the interpreted world and encompasses potential design solutions. Cognitive processes express the navigation from one world to another: interpretation, constructive memory, focus and action. This model offers a mechanistic view of reflection-in-action (Schön, 1983; Gero and Kannengiesser, 2008).

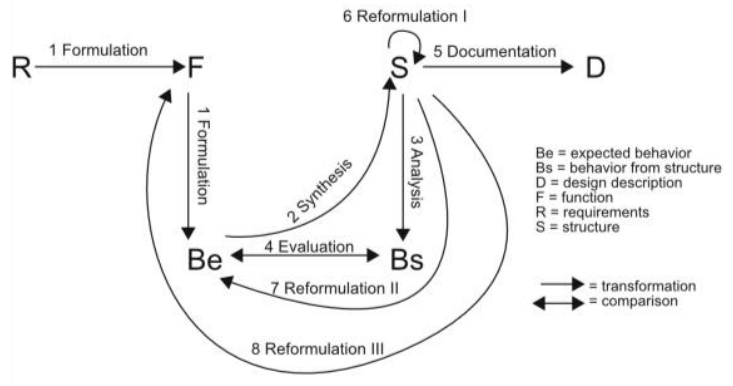

(a)

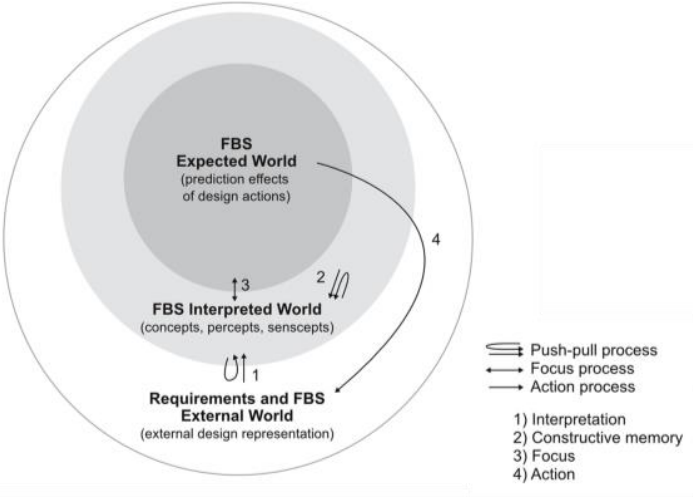

(b)

Figure 1. (a) FBS ontology based on Gero (1990), (b) Situated design framework based on Gero and Kannengiesser (2004)

\subsubsection{The situated FBS co-design model}

The situated FBS co-design model is a cognitive articulation of the FBS ontology that shows how ontological co-design processes are mapped onto the situated FBS model (Gero and Milovanovic, 2019), Figure 2.

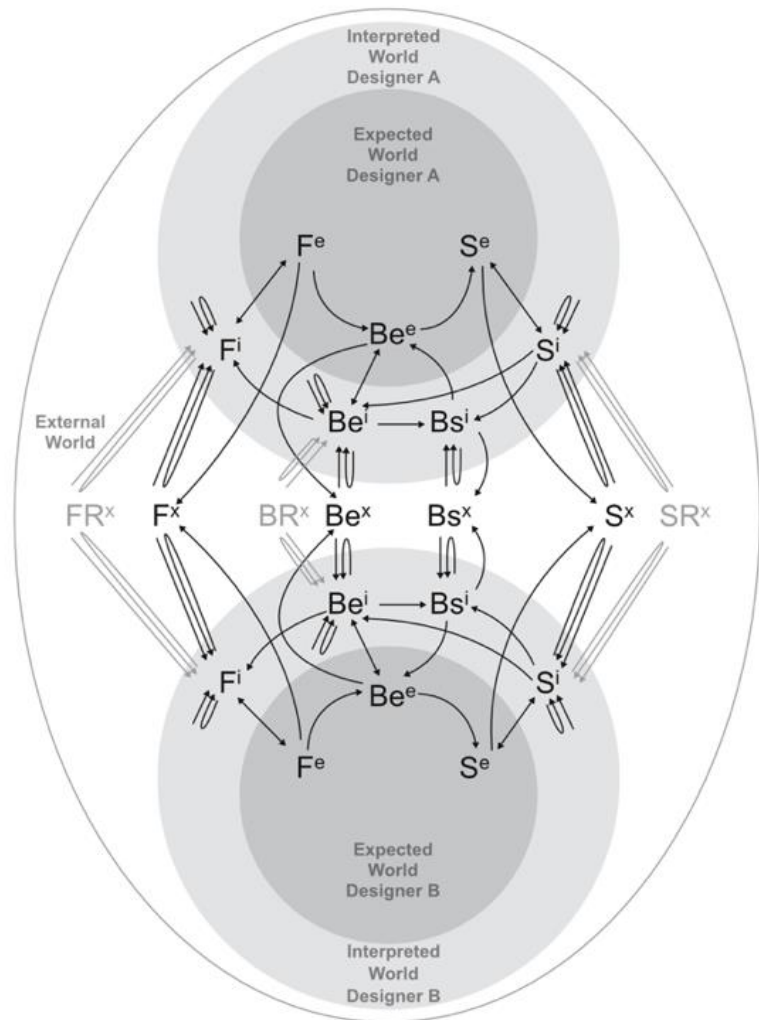

Figure 2. Situated FBS co-design model (Gero and Milovanovic, 2019) 
The FBS ontology describes design knowledge and design processes, therefore its co-design extension describes co-designing at a design-task level. The model is commutative, therefore all possible coconstructed situated FBS processes initiated by designer B and carried on by designer A can be represented symmetrically. Design described as a reflective practice (Schön, 1983) is both the learning objective and the pedagogic strategy supported by a learning by doing approach in the design studio. In it, design reflective practice is either demonstrated, explained, observed or accompanied. We use the situated FBS co-design model (Gero and Milovanovic, 2019) to represent the mentored reflective practice through a set of co-design processes.

\subsection{Methodology and data description}

To analyze design critiques, we used the protocol analysis method (Ericsson and Simon, 1984; Van Someren et al., 1994). The methodology of protocol analysis aims to study the cognitive processes of expert designers by analyzing their verbal statements. The protocol can be the transcription of a verbalization of a single designer (think-aloud protocol) or the transcription of the conversation of a group of designers (discussion protocol) (Jiang and Yen, 2009). In our case, we used the video recording of the critiques between tutors and students as video protocols. The verbalizations are segmented and each segment is associated with a code that refers to our theoretical framework. We used two coding schemes for our analysis. The first relates to design processes, coded based on the tutors' and students' conversations, while the second explores the manipulation of design representations, coded based on their actions and gestures recorded on the videos. To analyze the design processes, we coded our protocols with the FBS design issues. From coded protocols, we extracted design processes by looking at design issue syntactic transitions (Kan and Gero, 2017). The second coding scheme includes four potential actions on design representations: to sketch a design element, to model a design element, to point at a design element (deictic gesture) and to represent a design element or concept with a hand gesture.

The case study is the Sensory Architecture master studio at authors' university. Three students were followed over a period of three weeks. The sessions took place in the second half of the semester, after the validation of the design concept. For this studio, the design requirements were to integrate public equipment into a housing complex, and to take into account the environmental quality of the project. During the critiques observed, students use concept mock-ups they had previously developed as well as a set of other representations like plans, sections, perspectives drawings. Students are referred to as Student A, B and C in the rest of this paper and the Student A_1 label corresponds to the first review observed for this student, and so on.

\section{Results}

\subsection{Dominance of FBS design processes}

For each of the nine critiques analyzed, we measured the distribution of each FBS design processes, Table 1, to better understand the design activity during the critiques. For all critiques, except Student C's 2nd one, the Analysis and Reformulation 1 processes are dominant and represent at least $50 \%$ of all design processes formulated during the review. These processes are situated in the solution space, which shows the prevalence of cognitive effort placed on the design solution rather than on the design problem. The distribution of Reformulation 1 design processes increases between the first and third critiques, with an average of $26.5 \%(\mathrm{SD}=1.1)$ for critique $1,29.2 \%(\mathrm{SD}=3.2)$ for critique 2 and $36.6 \%$ $(\mathrm{SD}=5.4)$ for review 3 . The distribution of Analysis processes decreases slightly from critique $1(\mathrm{M}=$ $23.9 \%, \mathrm{SD}=1.6)$ to critique $2(\mathrm{M}=21.7 \%, \mathrm{SD}=5.5)$ and increases during the third critique $(\mathrm{M}=$ $30.9 \%, \mathrm{SD}=5.2$ ). The distribution of the Synthesis, Evaluation and Reformulation 2 processes is similar for critiques 1 and 2 . For the first two critiques these processes represent respectively about $15 \%, 16 \%$ and $13 \%$ of the total design processes. During the last critique, the average distribution of these processes decreases to $8.9 \%(\mathrm{SD}=4.2)$ for Synthesis processes, $13.1 \%(\mathrm{SD}=5.2)$ for Evaluation processes and $7.2 \%$ ( $\mathrm{SD}=2.9$ ) for Reformulation 2 processes. The Formulation and Reformulation 3 processes represent less than $4 \%$ of all design processes for each critique. The Formulation processes tend to decrease from critique to critique while the Reformulation 3 processes tend to slightly increase. 
Table 1. Standardized distribution (\%) of FBS design processes for each critique

\begin{tabular}{lccccccc} 
& Form. & Synth. & Analysis & Eval. & Ref. 1 & Ref. 2 & Ref.3 \\
\hline \hline Student A_1 & 1.3 & 17.5 & 23.5 & 12.8 & 27.4 & 16.2 & 1.3 \\
Student A_2 & 0.7 & 15.6 & 22.0 & 15.8 & 29.9 & 13.8 & 2.2 \\
Student A_3 & 1.8 & 9.5 & 29.4 & 17.6 & 32.1 & 6.8 & 2.7 \\
\hline Student B_1 & 1.9 & 18.1 & 22.6 & 15.5 & 26.8 & 13.6 & 1.5 \\
Student B_2 & 0.9 & 12.9 & 27.0 & 15.1 & 32.1 & 10.1 & 1.9 \\
Student B_3 & 1.0 & 4.4 & 36.8 & 7.4 & 42.6 & 4.4 & 3.4 \\
\hline Student C_1 & 3.8 & 11.5 & 25.7 & 19.9 & 25.3 & 11.5 & 2.3 \\
Student C_2 & 3.1 & 18.2 & 16.1 & 18.5 & 25.7 & 16.1 & 2.4 \\
Student C_3 & 0.7 & 12.7 & 26.7 & 14.2 & 35.0 & 10.3 & 0.5 \\
\hline Mean critique 1 & 2.3 & 15.7 & 23.9 & 16.1 & 26.5 & 13.8 & 1.7 \\
SD review 1 & 1.3 & 3.7 & 1.6 & 3.6 & 1.1 & 2.4 & 0.5 \\
\hline Mean critique 2 & 1.6 & 15.5 & 21.7 & 16.5 & 29.2 & 13.3 & 2.2 \\
SD review 2 & 1.3 & 2.6 & 5.5 & 1.8 & 3.2 & 3.0 & 0.3 \\
\hline Mean critique 3 & 1.2 & 8.9 & 30.9 & 13.1 & 36.6 & 7.2 & 2.2 \\
SD review 3 & 0.6 & 4.2 & 5.2 & 5.2 & 5.4 & 2.9 & 1.5 \\
\hline \hline
\end{tabular}

\subsection{Role of participants in formulating design processes}

The general distribution of design processes gives an overall description of the design activity during the critique. To better understand the contribution of each participant to the design critique, we conducted a correspondence analysis between the average distribution of the dominant FBS design processes and four participant's interactions: student to student (S), student to tutor (Student > tutor), tutor to student (Tutor > student) and tutor to tutor (T). Each review is represented on a separate graph, Figure 3.

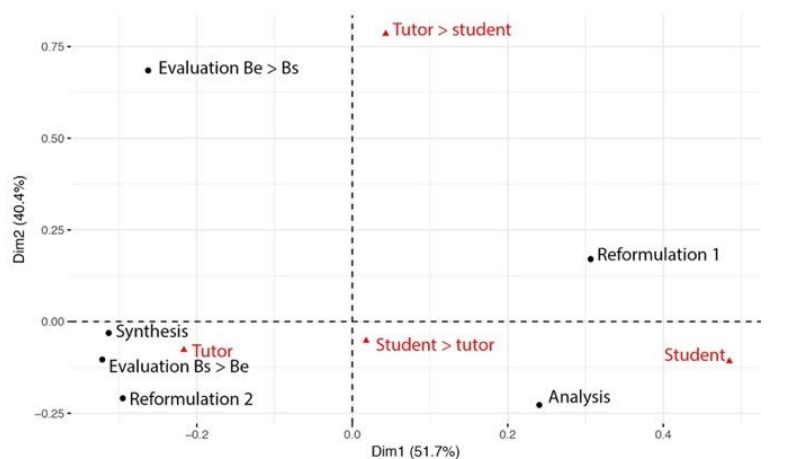

(a)

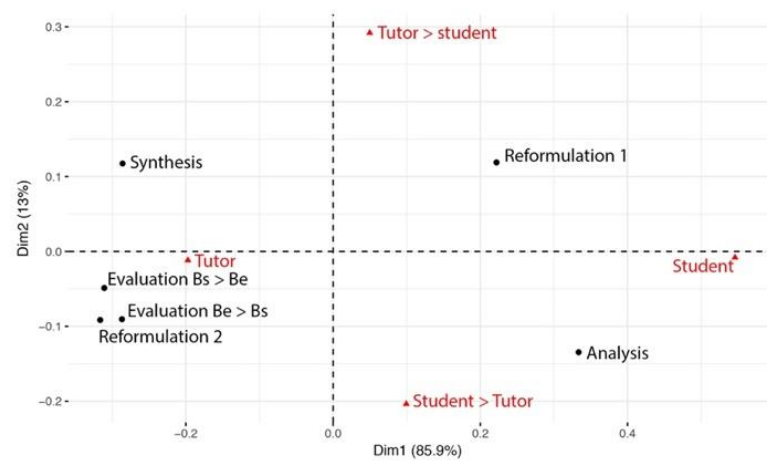

(b)

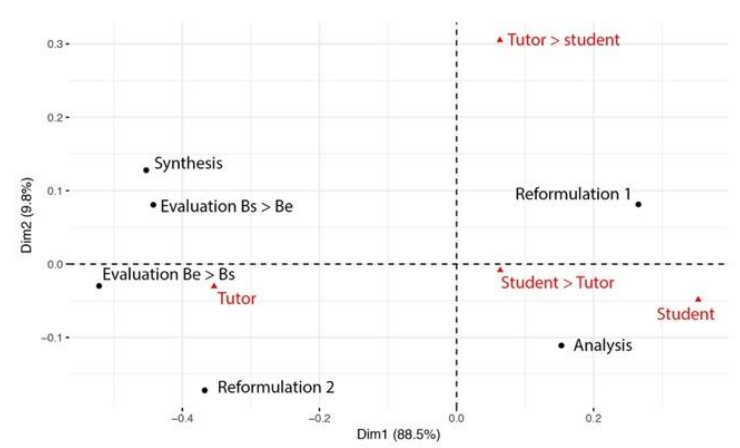

(c)

Figure 3. Correspondence analysis showing associations between participants' interactions and design processes: (a) critique 1 , (b) critique 2, (c) critique 3 
The four interactions have similar relative positions for all three critiques. This implies that tutor to student and student to tutor interactions have similarities in one dimension but are different in another dimension. Similarly, these results imply that tutor to tutor and student to student interactions have similarities in one dimension but are different in another dimension. Note that the quadrants these interactions sit in are different. We also see that Synthesis, Evaluation and Reformulation 2 processes appear on one side of the first dimension with the tutor interaction while Analysis and Reformulation 1 processes appear on the other side of this dimension. Related to co-design processes, the tutor to student interaction always appears in the same quadrant as the Reformulation 1 process, while the student to tutor one always appears in the same quadrant as the Analysis process.

\subsection{Modeling co-design processes}

The correspondence analysis reveals relative differences between the design processes formulated by the tutor and student during the design reviews. We used the situated FBS co-design model to represent qualitatively the role of each participant in the co-design activity, Figure 4.

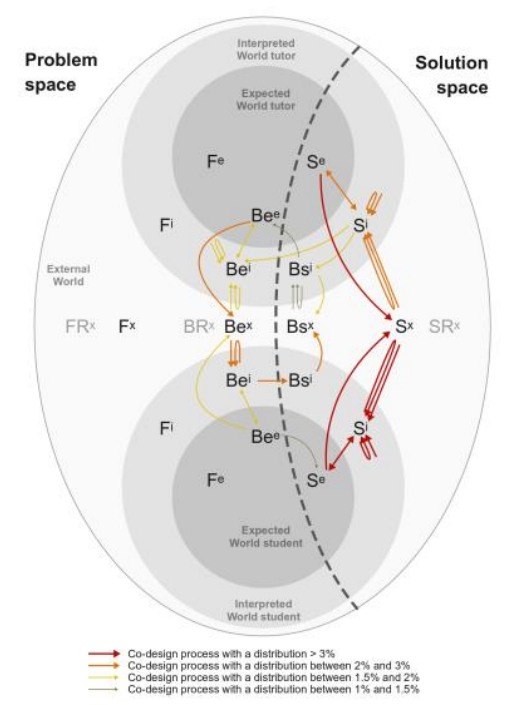

(a)

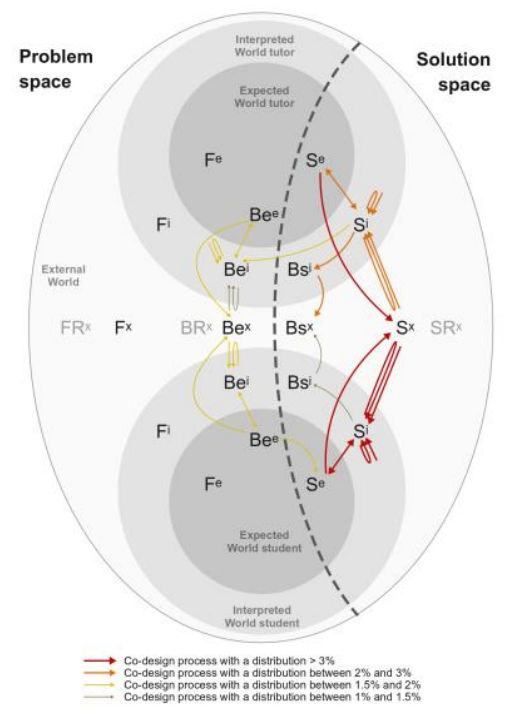

(b)

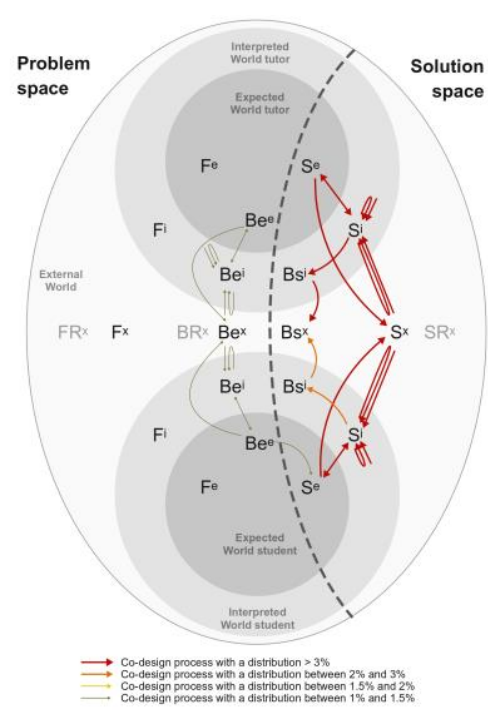

(c)

Figure 4. Representation of co-design processes: (a) critique 1, (b) critique 2 and (c) critique 3

The FBS ontology accounts for a distinction between the design problem space and the design solution space. Requirements (R), Function (F), and expected Behavior (Be) relate to the problem space whereas Structure (S) and Behavior from structure (Bs) relate to the problem space. We can represent both problem and solution spaces in the situated FBS co-design model, in order to explore where participants put their cognitive effort, on the design problem or the design solution. In our cases, co-design processes change slightly from critique to critique. In the first and second critiques, Figures 4(a) and (b), the cognitive effort is balanced between the problem space and the solution space. In the last critique, the focus is mainly on the design solution, Figure 4(c). We see that the temporality of the critiques has an effect on its focus.

\subsection{Manipulation of design representations}

The second element we looked at is participants' interactions with design representations. We quantified participants' type of actions on design representations, Figure 5. Design representations are used concurrently with the verbalization of a design activity in $60 \%$ of the cases, on average. The action of pointing at one representation largely dominates the others. Six percent of the verbalizations are accompanied by a gesture to represent a design element. In $4 \%$ of cases, the verbalizations are formulated while sketching, and in $2 \%$ of cases, they are associated with the modeling of the mock-up. Modeling appeared in four of the nine critiques to test new design solutions. These last three actions have appeared in our protocols but are not dominant. They are concurrent with a verbalization for about $12 \%$ of the cases. 


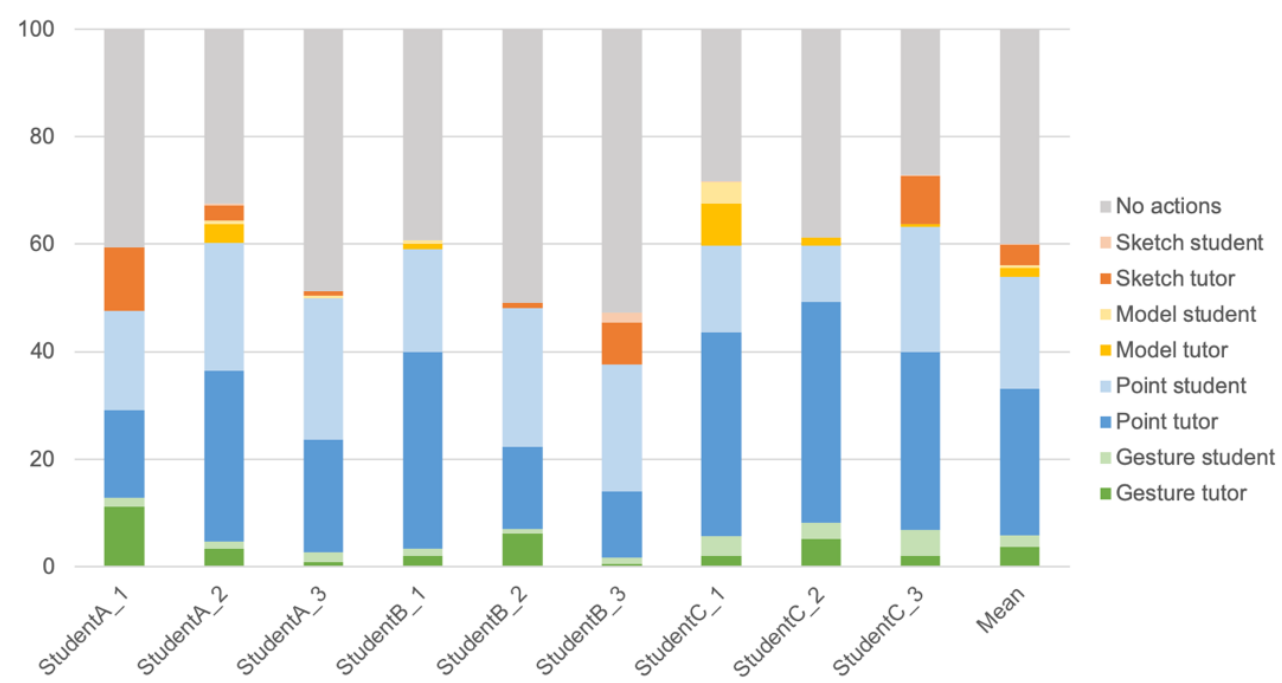

Figure 5. Standardized distribution of actions on design representations for all sessions

\subsection{Relation between actions on design representations and design processes}

The correspondence analysis, Figure 6, illustrates proximities between dominant FBS design processes and actions on design representations. The action of modeling is not associated with any FBS design processes in our dataset. Two of the actions are situated on one side of dimension 1 (on the $\mathrm{x}$-axis), sketching and representing with a gesture, while pointing is on the other side of this dimension. Sketching and representing by a gesture are situated in two different quadrants of the graph. This shows relative differences in the correspondence between the FBS design process and the actions on design representations. Pointing is associated with Evaluation processes, sketching with Analysis and Reformulation 1 processes, while representing by a gesture appears in the same quadrant as Synthesis and Reformulation 1 processes.

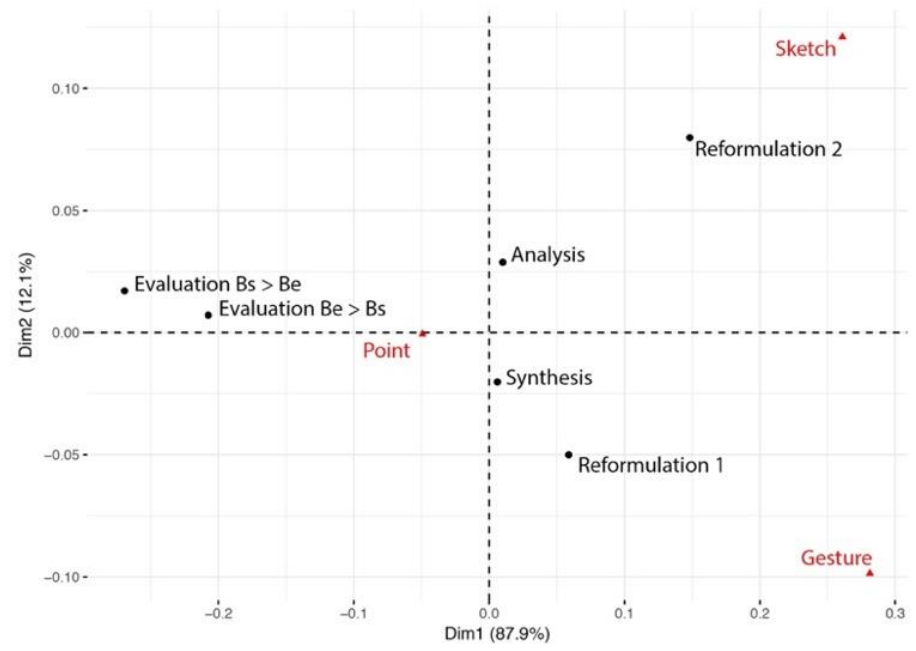

Figure 6. Correspondence analysis between dominant design process and actions on design representations

\section{Discussion}

The methodology used provides a way to analyze our dataset, to compare our results by critique sessions and to point to trends concerning cognitive behaviors of students and tutors during these critiques. In situ observation has its shortcoming as social factors influences the situation. Other limitations of our case study are the small sample size and that the length of each critique varies. The intent of the tutor during the critiques is to place the student in several situations in relation to the 
design activity: active, reflective and collaborative. The student usually initiates the critique by presenting their design, and there follows a discussion between the tutor and the student on the design presented. The tutor may ask for clarifications on an element, ask questions to stimulate reflection, point out problematic elements and sometimes suggest a partial or total solution to a problem highlighted. The results from our analysis correspond to the role described. Tutors' interventions tend to be associated with Evaluation, Reformulation 2 and Synthesis processes. Tutors are the ones formulating processes that are associated with design space navigation processes. Evaluation and Reformulation 2 processes illustrate processes to reflect and point out problematic design elements. Synthesis processes can be assimilated to the formalization of a design solution. The synthesis of a solution serves as a primary generator (Darke, 1979) to reduce the problem space. Students often struggle to synthesize the elements they get from their site analysis into a design concept. The role of the tutor is to guide the students in this task so that they acquire this skill of going back and forth between the requirements and the formalized design concept. The iterative dimension of the design process involves questioning the design proposal, which is led by tutors. The formulation processes by students are associated with the Analysis processes for all reviews, which is consistent with the action of presenting and explaining their design.

About $60 \%$ of the verbalizations during the reviews are concurrent with an action on design representations. The actions of gesture, pointing to an element and representing an element, are concurrent with half of the reviews' verbalizations. The action of pointing a representation is largely dominant over the other actions and is used to refer to a design element. The referencing process by pointing favors the development of the common ground between participants (Defays et al., 2014). This action implies that participants agree on the element that is being discussed. When tangible design representations (plans, sections, elevations, mock-ups) are not enough to illustrate what is verbalized, participants use gestures or sketches, often to represent a new design element or explain a concept. The use of gestures to represent an element or concept and the action of sketching is much less frequent but appeared in each critique. According to Détienne et al. (2006), the action of sketching tends to be associated with solution-generation activities while the action of showing (pointing) corresponds to interpretation or information actions. Contrary to our expectations, in our case studies, the action of sketching tends to be associated with Reformulation 2 processes more than with Synthesis processes. Pointing, on the other hand, is associated with Evaluation processes, which is consistent with the notion of interpretation, and which is formalized in our case by the comparison between design intentions and the current design state.

\section{Conclusion}

In this paper, we explored and described a case study of nine design critiques within the studio pedagogy using the mentored reflexive practice framework based on the FBS ontology. Studying design critiques is of particular interest as the studio pedagogy is being increasingly used in disciplines such as engineering design and software design that have not previously used it as a pedagogic paradigm. A more complete understanding can only be obtained with a larger cohort of students than studied here. However, the measurement processes used here (Kan and Gero, 2017) can provide a quantitative basis for the role of design critiques within the studio pedagogy. The results presented provide a model of design studio pedagogy that can serve as a baseline to compare the effect of other types of design studio critiques that use alternative type of design representations (digital media), or alternative types of studio format (peer review, group critiques). Testing and refining design studio pedagogy is necessary to provide students with suitable learning experiences so that they can acquire skills and knowledge that they will build upon as practitioners.

\section{References}

Barrett, T. (2000), "Studio Critiques of Student Art: As They Are, as They Could Be with Mentoring”, Theory Into Practice, Vol. 39 No. 1, pp. 29-35.

Cardoso, C. et al. (2014), "Question asking in design reviews: How does inquiry facilitate the learning interaction?", Design Thinking Research Symposium 10, Purdue University.

Cross, N. (2006), Designerly Ways of Knowing, Springer, London. 
Darke, J. (1979), “The primary generator and the design process", Design studies, Vol. 1 No. 1, pp. 36-44.

Defays, A., Jeunejean, A. and Giboin, A. (2014), "Impact de la médiatisation technologique sur la mobilisation des modalités non-verbales dans les références demonstratives”, Symposium COMMON'14, 89-101. Liège, Belgium.

Dennen, V.P. and Burner, K.J. (2008), “The Cognitive Apprenticeship Model in educational Practice”, In: Spector, M.J., Merrill, D.M., van Merrienboer, J. and Driscoll, M.P. (Eds.), Handbook of Research on Educational Communications and Technology, Lawrence Erlbaum Associates, Mahwah, NJ, USA, pp. 813-828.

Détienne, F., Visser, W. and Tabary, R. (2006), "Articulation des dimensions graphico-gestuelle et verbale dans l'analyse de la conception collaborative", In Language et cognition: Contraintes pragmatiques. Psychologie de l'interaction, L'Harmattan, pp. 283-307.

Dong, A., Garbuio, M. and Lovallo, D. (2016), "Generative sensing in design evaluation”, Design Studies, Vol. 45, pp. 68-91.

Ericsson, K.A. and Simon, A.H. (1984), Protocol Analysis: Verbal Reports as Data, MIT Press.

Estevez, D. (2001), Dessin d'architecture et inforgraphie: L'évolution contemporaine des pratiques graphiques (CNRS Edition).

Ferguson, E.S. (1992), Engineering and the Mind's Eye, MIT Press.

Ferreira, J., Christiaans, H. and Almendra, R. (2016), "A visual tool for analysing teacher and student interactions in a design studio setting", CoDesign, Vol. 12 No. 1-2, pp. 112-131.

Gero, J.S. (1990), "Design prototypes: a knowledge representation schema for design”, AI Magazine, Vol. 11 No. 4, pp. 26-36.

Gero, J. S. and Jiang, H. (2016), "Exploring the Design Cognition of Concept Design Reviews Using the FBSbased Protocol Analysis", In: Adams, R. S. and Siddiqui, J. A. (Eds.), Analyzing Design Review Conversations, Purdue University Press, p. 177.

Gero, J.S. and Kannengiesser, U. (2004), "The situated function-behaviour-structure framework", Design Studies, Vol. 25 No. 4, pp. 373-391.

Gero, J.S. and Kannengiesser, U. (2008), “An ontological account of Donald Schön's reflection in designing”, International Journal of Design Sciences and Technologies, Vol. 15 No. 2, pp. 77-90.

Gero, J.S. and Milovanovic, J. (2019), "The situated function-behavior-structure co-design model", CoDesign.

Goldschmidt, G. et al. (2014), “Three studio critiquing cultures: Fun follows function or function follows fun?", Design Thinking Research Symposium 10, Purdue University.

Goldschmidt, G., Hochman, H. and Dafni, I. (2010), "The design studio "crit": Teacher-student communication", Artificial Intelligence for Engineering Design, Analysis and Manufacturing, Vol. 24 No. 03, pp. 285-302.

Healy, J. (2016), "The Components of the "Crit" in Art and Design Education", Irish Journal of Academic Practice, Vol. 5 No. 1, p. 7.

Heylighen, A., Bouwen, J.E. and Neuckermans, H. (1999), "Walking on a thin line: Between passive knowledge and active knowing of components and concepts in architectural design", Design Studies, Vol. 20 No. 2 , pp. 211-235.

Jiang, H. and Yen, C.-C. (2009), "Protocol Analysis in Design Research: a Review", Design, Rigor \& Relevance, presented at the IASDR, Seoul, Korea, pp. 147-156.

Kan, J.W.T. and Gero, J.S. (2017), Quantitative Methods for Design Protocol Analysis, Springer.

Lawson, B. and Dorst, K. (2009), Design Expertise, Architectural Press., Routledge.

Lebahar, J.-C. (1983), Le dessin d'architecte. Simulation graphique et réduction d'incertitude (Parenthèses).

Oh, Y. et al. (2013), "A theoretical framework of design critiquing in architecture studios", Design Studies, Vol. 34, pp. 302-325.

Purcell, A.T. and Gero, J.S. (1998), "Drawings and the design process", Design Studies, Vol. 19 No. 4, pp. 389-430.

Schön, D. (1983), The Reflective Practitioner: How Professionals Think in Action, Temple Smith, London.

Schön, D. (1992), "Designing as reflective conversation with the materials of a design situation", Research in Engineering Design, Vol. 3 No. 3, pp. 131-147.

Schön, D.A. (1985), The Design Studio, RIBA, London.

Van Someren, M.W., Barnard, Y.F. and Sandberg, J.A.C. (1994), The Think Aloud Method: A Practical Guide to Modelling Cognitive Processes, Academic Press, London.

Wolmarans, N. (2016), "Inferential reasoning in design: Relations between material product and specialised disciplinary knowledge”, Design Studies, Vol. 45, pp. 92-115. 